Research Paper

\title{
Repression of FGF signaling is responsible for Dnmt3b inhibition and impaired de novo DNA methylation during early development of in vitro fertilized embryos
}

\author{
Wei Fu*, Yuan Yue*, Kai Miao, Guangyin Xi, Chao Zhang, Wenjuan Wang, Lei An, Jianhui Tian ${ }^{\bowtie}$ \\ National Engineering Laboratory for Animal Breeding; Key Laboratory of Animal Genetics, Breeding and Reproduction of the Ministry of Agriculture; College \\ of Animal Science and Technology, China Agricultural University, No.2 Yuanmingyuan West Road, Beijing 100193, P. R. China. \\ *These authors contributed equally to this work. \\ $\bowtie$ Corresponding author: Jianhui Tian. E-mail: tianjh@cau.edu.cn.
}

(C) The author(s). This is an open access article distributed under the terms of the Creative Commons Attribution License (https://creativecommons.org/licenses/by/4.0/). See http://ivyspring.com/terms for full terms and conditions.

Received: 2020.08.06; Accepted: 2020.09.12; Published: 2020.10.03

\begin{abstract}
Well-orchestrated epigenetic modifications during early development are essential for embryonic survival and postnatal growth. Erroneous epigenetic modifications due to environmental perturbations such as manipulation and culture of embryos during in vitro fertilization (IVF) are linked to various shortor long-term consequences. Among these, DNA methylation defects are of great concern. Despite the critical role of DNA methylation in determining embryonic development potential, the mechanisms underlying IVF-associated DNA methylation defects, however, remains largely elusive. We reported herein that repression of fibroblast growth factor (FGF) signaling as the main reason for IVF-associated DNA methylation defects. Comparative methylome analysis by postimplantation stage suggested that IVF mouse embryos undergo impaired de novo DNA methylation during implantation stage. Further analyses indicated that Dnmt3b, the main de novo DNA methyltransferase, was consistently inhibited during the transition from the blastocyst to postimplantation stage (Embryonic day 7.5, E7.5). Using blastocysts and embryonic stem cells (ESCs) as the model, we showed repression of FGF signaling is responsible for Dnmt3b inhibition and global hypomethylation during early development, and MEK/ERK-SPI pathway plays an essential mediating role in FGF signaling-induced transcriptional activation of Dnmt $3 b$. Supplementation of FGF2, which was exclusively produced in the maternal oviduct, into embryo culture medium significantly rescued Dnmt3b inhibition. Our study, using mouse embryos as the model, not only identifies FGF signaling as the main target for correcting IVF-associated epigenetic errors, but also highlights the importance of oviductal paracrine factors in supporting early embryonic development and improving in vitro culture system.
\end{abstract}

Key words: in vitro fertilization, DNA methylation, Dnmt3b, FGF signaling

\section{Introduction}

IVF is one of the most effective and successful assisted reproductive technologies for treating infertility that affects $\sim 15 \%$ of couples globally. Currently, it is estimated that more than 9 million babies have been born worldwide since the first IVF baby was born in 1978, and IVF contributes to $1-5 \%$ of all newborns in developed countries [1]. Although the great majority of IVF-conceived offspring are in good health, increasing epidemiologic analyses in humans and laboratory studies in animals show that IVF is associated with various short- or long-term consequences, including pregnancy complications, preterm birth, low birth weight, birth defects [2-6], as well as higher disease risks in later life, such as heart disease, diabetes, and hypertension [7-11].

Mammalian early development is controlled by a series of spatiotemporally regulated epigenetic modifications that are critical for acquiring developmental potential. In particular, from preimplantation to postimplantation stage, 
environmental perturbations such as manipulation and culture of embryos in vitro, can affect the epigenetic programming of the developing embryos, and thus leading to erroneous epigenetic modifications, which are expected to be linked to those various short- or long-term consequences in IVF embryos and offspring [11-13]. Among the IVF-associated epigenetic errors, DNA methylation defects are extensively studied. Earlier studies that reported imprinting disorders in IVF-conceived Angelman (AS) and Beckwith-Wiedemann syndromes (BWS) offspring [14-16], have raised safety concerns of clinical use of IVF. After that, imprinting loss of specific loci, as well as globally altered methylome, have been reported repeatedly in IVFconceived embryos and offspring in humans [17, 18], domestic animals [19-21], and mouse models [22-25]. Despite the critical role of DNA methylation in determining embryonic development and postnatal growth, the mechanisms underlying IVF-associated DNA methylation defects, however, remains largely elusive. Thus, there has never been potential strategy for preventing or correcting those disturbances.

Using methylated DNA immunoprecipitationsequencing (MeDIP-seq) data that profiles DNA methylation maps from in vivo (IVO) and IVF mouse postimplantation embryos, which have established static DNA methylation patterns following de novo DNA methylation [26, 27], we found IVF processes induced global hypomethylation in postimplantation embryos. This finding suggests an impaired de novo DNA methylation, the critical epigenetic event that is prerequisite for re-establishing the DNA methylation patterns from a globally hypomethylated state in blastocysts to relatively static DNA methylation patterns in postimplantation embryos. Given the DNMT3B is the main enzyme required for de novo DNA methylation during early implantation [26, 28], we have explored the mechanism underlying impaired de novo DNA methylation by focusing on the expression patterns of Dnmt3b in IVF embryos, as well as the upstream signaling responsible for transcriptional regulation of Dnmt3b. Our study identifies repression of FGF signaling as one of the main barriers that induces DNA methylation defects in IVF postimplantation embryos, and provides a promising approach for preventing or correcting IVF-associated epigenetic errors.

\section{Materials and Methods}

\section{Mouse embryo preparation and collection}

All experimental procedures were approved by and performed in accordance with the guidelines of the Institutional Animal Care and Use Committee of
China Agricultural University. Crlj:CD1 (ICR) mice used in present study were provided by SPF Biotechnology Co., Ltd (Beijing, China). Female mice were superovulated by an i.p. injection of $5 \mathrm{IU}$ pregnant mare serum gonadotrophin (PMSG, Ningbo Hormone Product Co., Ltd, Ningbo, China) and a further i.p. injection $48 \mathrm{~h}$ later of $5 \mathrm{IU}$ human chorionic gonadotropin (hCG, Ningbo Hormone Product Co., Ltd) after $48 \mathrm{~h}$. For IVF, $12 \mathrm{~h}$ after hCG injection, mature oocytes were collected from the ampullae at $14 \mathrm{~h}$ post-hCG treatment, and in vitro fertilized by spermatozoa separated from ICR male epididymis. Then, zygotes were washed and cultured to the blastocyst stage in potassium simplex optimization medium containing amino acids (KSOM+AA, Millipore, Darmstadt, Germany) at $37^{\circ} \mathrm{C}$ in 5\% CO2 under $20 \%$ atmospheric oxygen concentration. To obtain IVO embryos, superovulated ICR female mice were cocaged individually with ICR males after the hCG injection.

The collection of IVO and IVF embryos was performed based on the well-established procedures and methods of our own published studies [13, 29-31]. For preimplantation embryo collection, IVO embryos at the 8-cell, and morula stages (68-70, and 78-80 h after hCG, respectively) were recovered from donors by flushing the oviduct with M2 medium (formulation is shown in Table S7). The IVF blastocysts were harvested at 106-112 $\mathrm{h}$ post-hCG after culturing in KSOM medium, while control IVO blastocysts were harvested at $96-100 \mathrm{~h}$ post-hCG. In each group, well-developed embryos of similar morphology were selected for further analysis or embryo transfer.

For treatment with FGF2 or MEK/ERK inhibitor during in vitro culture, exogenous FGF2 (R\&D, Minneapolis, MN, USA) or $5 \mu \mathrm{M}$ MEK/ERK inhibitor PD0325901 (PD, Selleck, Houston, TX, USA) were supplemented into in vitro culture medium from the 4-cell stage, and embryos were collected at blastocyst stage for further analyses.

For postimplantation embryo collection, pseudopregnant ICR mice were cocaged individually with vasectomized with males $3.5 \mathrm{~d}$ before embryo transfer. Well-developed blastocysts with similar morphologies were selected for embryo transfer. Twelve blastocysts were transferred to each recipient, with six embryos in each uterine horn. E7.5 or E10.5 decidua were separated from uterus and fetuses, and embryonic parts of the E7.5 and E10.5 embryos were separated and washed by PBS before further analyses.

\section{High-throughput RNA sequencing (RNA-seq) and bioinformatic analysis}

RNA-seq and bioinformatic analysis were 
performed based on the processes used in our previously published studies [31, 32]. In the present study, 38394, 795 and 278 embryos in the IVF groups and 37271,822 and 324 embryos in the IVO groups were sampled at E3.5, E7.5 and E10.5, respectively. Embryos at different stages were washed by ice-cold PBS for twice and cryopreserved into ultra-low temperature freezer till use. Total RNAs were extracted with TRIzol Reagent (Invitrogen, Carlsbad, CA, USA). Polyadenylated RNAs were isolated using the Oligotex mRNA Midi Kit (Qiagen, Valencia, CA, USA). The RNA-seq libraries were constructed using the SOLiD Whole Transcriptome Analysis Kit following the standard protocol (AB, Applied Biosystems, USA) and sequenced on the Applied Biosystems SOLiD platform to generate high-quality single-end reads. The raw reads were aligned to genome sequences, trimming off a nucleotide each from the $5^{\prime}$ and $3^{\prime}$ ends and allowing up to two mismatches. Reads mapped to multiple locations were discarded and only uniquely mapped reads were used for the subsequent analysis [13, 31, 32]. Briefly, 73,107,951, 44,038,064 and 41,536,756 uniquely mapped reads in IVO embryos at E3.5, E7.5 and E10.5 were obtained with $39.83 \%, 46.88 \%$ and $47.07 \%$ mapping rate. Furthermore, 84,623,384, 40,616,266 and 45,876,283 uniquely mapped reads in IVF embryos at E3.5, E7.5 and E10.5 were obtained with $46.14 \%, 47.25 \%$ and $48.94 \%$ mapping rate. Gene expression levels were measured in reads per kilobase of exon model per million mapped reads (RPKM). Of note, due to the small amount of nucleic acid in each early embryo, we used a single pooling strategy to perform RNA-seq, thus, results in the present study did not have error bars [33, 34].

The Database for Annotation, Visualization and Integrated Discovery (DAVID v6.7; http://david. abcc.ncifcrf.gov) was used to annotate biological themes (Gene ontology, GO). The Kyoto Encyclopedia of Genes and Genomes (KEGG; http:/ / www.genome. $\mathrm{jp} / \mathrm{kegg} /$ ) was used to determine the associated pathways. Phenotype annotations of differentially expressed genes were analyzed based on the Mouse Genome Informatics (MGI; http:/ / www.informatics. jax.org/phenotypes.shtml) database.

\section{Methylated DNA immunoprecipitation- sequencing (MeDIP-seq)}

In the present study, the same embryos were used to perform both RNA-seq and MeDIP-seq, which were demonstrated in our previous studies [32, 35]. Briefly, Embryo samples were rinsed by PBS for twice and cryopreserved into ultra-low temperature freezer till use. Global genomic DNA, including both nuclear DNA (nDNA) and mitochondrial DNA (mtDNA), was isolated from embryo samples using Qiagen DNeasy kits (Qiagen), according to the modified manufacturer's instructions. The quality of each DNA sample was analyzed for integrity, purity and concentration on a Nanodrop Spectrophotometer DN-1000 (Nanodrop Technologies, Wilmington, DE, USA). Genomic DNA was then fragmented using a Covarias sonication system (Covarias, Woburn, MA, USA). After sonication, the fragments were denatured to produce single-stranded DNA (ssDNA). Following denaturation, the ssDNA was incubated with monoclonal 5-methyl-cytidine (5-mC) antibodies (Biorad, Minneapolis, MN, USA) at room temperature for $1 \mathrm{~h}$. Magnetic beads conjugated to anti-mouse-IgG were then used to bind the anti-5-mC antibodies, and unbound DNA was removed along with the supernatant. Finally, $10 \mathrm{mg} / \mathrm{ml}$ proteinase $\mathrm{K}$ was used to digest the antibodies $\left(60^{\circ} \mathrm{C} 45 \mathrm{~min}, 95^{\circ} \mathrm{C} 15\right.$ min), and the DNA was released and collected. Sequencing was carried out on the Illumina Hiseq 2000 (Illumina, San Diego, CA, USA) following the standard protocol to generate paired-end $50 \mathrm{bp}$ reads by a commercial company (BGI, Shenzhen, Guangdong, China) [32, 35]. Briefly, 84,075,585 and $86,570,768$ uniquely mapped reads were obtained in IVO and IVF embryos at E7.5 with 51.50\% and 53.02\% mapping rates. Furthermore, 83,183,633 and $81,718,963$ uniquely mapped reads were obtained in IVO and IVF embryos at E10.5 with $50.95 \%$ and $50.05 \%$ mapping rates. DNA methylation were measured in RPKM.

\section{Mouse ES cell culture and differentiation}

Mouse ESCs line PGK12.1 was used in the present study. Cells were cultured in stem cell medium (SCM) containing Knockout DMEM base medium (Invitrogen), 10\% fetal bovine serum (Hyclone, Logan, UT, USA), $1 \times$ non-essential amino acids (Millipore), $2 \mathrm{mM}$ GlutaMAX (Invitrogen), 55 $\mu \mathrm{M} \quad \beta$-mercaptoethanol (Invitrogen), $1 \times 10^{5}$ units leukemia inhibitory factor (LIF, Invitrogen) and $1 \%$ penicillin-streptomycin (Invitrogen), and incubated in a humidifier containing $5 \% \mathrm{CO}_{2}$ at $37^{\circ} \mathrm{C}$. To induce differentiation, $3.0 \sim 5.0 \times 10^{3} / \mathrm{cm}^{2}$ cells were planted onto glasses coated by $5 \mu \mathrm{g} / \mathrm{cm}^{2}$ fibronectin in SCM. After $24 \mathrm{~h}$ growth, cells were rinsed by $1 \mathrm{ml}$ PBS and differentiation medium, same as SCM without LIF, was added into each well to induce cell differentiation.

\section{Quantitative real-time polymerase chain reaction ( $q R T-P C R)$}

Embryos or cells used for RNA extraction were washed by PBS for twice and transferred into $1.5 \mathrm{ml}$ centrifuge tube, and then, $1 \mathrm{ml}$ TRIzol reagent were 
added. After $10 \mathrm{~min}$ incubation on ice, samples were cryopreserved into ultra-low temperature freezer till use. Total RNA was extracted from embryos or cells following the manufacturer's instructions with TRIzol reagent (Invitrogen). HiScript II Q Select RT SuperMix for qPCR kit (Vazyme, Nanjing, China) was used to reverse-transcribe $1 \mu \mathrm{g}$ total RNA into cDNA according to the manufacturer's protocol. Quantitative real-time PCR analysis was performed using SsoFast EvaGreen Supermix (Bio-rad). Primers used in present study were exhibited in Table S1. Gene relative expression was referenced to Gapdh and calculated by the $2^{-\Delta \Delta C t}$ method.

\section{Western blot}

Embryos and cells used to detect protein level were rinsed by PBS for twice, and collected into centrifuge tube on ice, and $30 \mu \mathrm{l} 2 \times$ lammli sample buffer (Bio-rad) with 5\% $\beta$-mercaptoethanol was added following $100{ }^{\circ} \mathrm{C}$ boiled for $10 \mathrm{~min}$. For differentiated cells, ice-cold PBS buffer was used to wash cells for three times, then RIPA buffer containing 1× protease cocktail (Beyotime Biotech. Inc., Shanghai, China) was added and cells were suspended and shake for several times on ice. Lysis solution was then centrifuged at $12500 \times \mathrm{g}$ for $10 \mathrm{~min}$ and total concentration of supernatant protein was measure by BCA protein quantification kit (Beyotime Biotech. Inc.). $12 \%$ sodium dodecyl sulfate polyacrylamide gel electrophoresis (SDS-PAGE) was used to separate total protein samples and transferred to a PVDF membrane (Millipore) following 5\% skim milk blocking for $1 \mathrm{~h}$ at room temperature. Primary antibody DNMT3B (1:200; GeneTex, Irvine, CA, USA) and $\beta$-tubulin (1:1000, Proteintech, Chicago, IL, USA) were diluted by blocking solution and the membranes were incubated overnight at $4{ }^{\circ} \mathrm{C}$. Then, membranes were rinsed by TBST buffer for three times, and incubated with secondary antibodies conjugated with HRP for $1 \mathrm{~h}$ at room temperature. After three washes with TBST buffer, target protein bands were visualized using eECL Western Blot Kit (CWbio., Beijing, China) and detected by 5200 Imaging system (Tanon, Shanghai, China).

\section{Immunofluorescence analysis}

$4 \%$ paraformaldehyde in PBS was used to fix embryos (overnight at $4{ }^{\circ} \mathrm{C}$ ) or cells $(20 \mathrm{~min}$ at room temperature) after culture medium removing. After three washes by PBS, samples were permeabilizing with $0.5 \%$ Triton-X 100 for $0.5 \sim 1 \mathrm{~h}$ at room temperature, and blocking with $1 \%$ BSA-PBS at $4{ }^{\circ} \mathrm{C}$ for 2 6 h. For 5-mC staining, before blocking, permeabilized embryos or cells should be treated with $4 \mathrm{M} \mathrm{Hcl}$ for $20 \mathrm{~min}$, and then neutralized with $100 \mathrm{Mm}$
Tris-Hcl $(\mathrm{pH}=8.0)$ for $30 \mathrm{~min}$. Next, diluted primary antibodies DNMT3B (1:200, GeneTex), CDX2 (1:200; BioGenex, Fremont, CA, USA), NANOG (1:1000, Abcam, Cambridge, MA, USA) or 5-mC (1:250, Bio-rad) was used to incubate with samples overnight at $4{ }^{\circ} \mathrm{C}$. After three washes by PBS, labeled secondary antibodies Alexa Fluor-488 (1:1000, Invitrogen) or Alexa Fluor-594 (1:1000, Invitrogen) was respectively added into samples under dark environment for $1 \mathrm{~h}$ at room temperature. Samples were then counterstained with DAPI, and imaged using an BX51 microscope (Olympus, Tokyo, Japan) accompanied with digital microscope camera (Olympus). All photographs were quantitated by Image J software (Rawak Software Inc., Stuttgart, Germany) and background subtraction was performed following the previous report [36].

\section{Chromatin immunoprecipitation (ChIP) assay}

Differentiated cells were cross-linked with 1\% formaldehyde and subjected to ChIP assay according to the protocols of ChromaFlash High-sensitivity ChIP kit (Epigentek, Farmingdale, NY, USA). The DNA lysate was crushed by ultrasonic breaker (Covarias) to produce 100 700 bps fragments. To obtain input controls, $1 \mu \mathrm{g}$ DNA in each group was released and purified, and diluted to 10 times before use. Primary antibody SP1 (Santa Cruz Biotech., Santa Cruz, CA, USA) and negative control non-immune IgG were used to precipitate binding DNA fragments. Quantitative PCR was performed and enrichment was calculated as follows: \% Input $=100 \times 2^{\text {(Ct (adjusted input) }}$ $\mathrm{Ct}(\mathrm{IP})), \mathrm{Ct}$ (adjusted input) = input $\mathrm{Ct}-3.32$. ChIP-qPCR primers were showed in Table S1.

\section{Fgf4 gene knockout}

CRISPR/CAS9n was used to knockout Fgf4 gene in PGK12.1. Using online guide designer Benchling, we acquired and synthesized small guide RNA (sgRNA). sgRNAs were constructed to $\mathrm{pSpCas9n(BB)-}$ 2A-Puro vector (PX462, purchased from Addgene) and transfected into PGK12.1 using Lipofectamine 2000 (Invitrogen) following the manufacturer's instructions. $24 \mathrm{~h}$ after transfection, $2 \mu \mathrm{g} / \mathrm{ml}$ puromycin was added into SCM to screen positive clones. Single clones were picked out after 5 days consistently screening, and PCR product sequencing was performed to identify knockout cell lines. All sgRNAs and PCR primers used for identification of knockout cell lines were showed in Table S1.

\section{Proliferation analysis}

EdU staining was used to measure the proliferation of embryos in the present study. Following the protocols of BeyoClick ${ }^{\mathrm{TM}}$ EdU-594 Cell Proliferation Kit (Beyotime Biotech. Inc.), $10 \mu \mathrm{M}$ EdU was added to KSOM medium and embryos were 
incubated at $37{ }^{\circ} \mathrm{C}$ for $2 \mathrm{~h}$. After fixation and permeabilization, embryos were incubated with the Click Reaction mixture for $2 \mathrm{~min}$ and stained with DAPI. BX51 microscope (Olympus) and Image J software (Rawak Software Inc.) were respectively used to photograph and quantitate EdU intensity in each group.

\section{Statistical analysis}

All experiments were replicated at least three times. Results were represented as means \pm Standard Error of Mean (SEM) and analyzed with ANOVA, the Student's test or correct $\chi^{2}$ using the SPSS version 18.0 software (IBM Corp., Armonk, NY, USA). The $p$-value below 0.05 was considered as the threshold of significant statistics.

\section{Results}

\section{IVF embryos exhibited global DNA hypo- methylation by postimplantation stage}

To test the effect of IVF process on DNA methylation patterns of postimplantation embryos, we compared our previously published global MeDIP-seq data of IVO and IVF embryos at E7.5 and E10.5 [31, 32, 35], which have established relatively static DNA methylation patterns through the process of de novo DNA methylation [26, 27]. We focused our analysis on promoter DNA methylation since promoters are main targets for transcriptional regulation of developmental genes in determining pluripotent or differentiated states [37, 38]. 822 IVO and 795 IVF embryos at E7.5, 324 IVO and 278 IVF embryos at E10.5 were respectively used to perform MeDIP-seq. Compared with that in IVO embryos, a higher proportion of promoters were hypomethylated in IVF postimplantation embryos (Fig. 1A). Among the hypomethylated promoters in IVF embryos, a substantial proportion showed moderate $(2<\mathrm{FC}<3$, $18.41 \%$ at E7.5 and $27.56 \%$ at E10.5) or dramatic (FC $>$ 3 or absent in IVF, $5.93 \%$ at E7.5 and $13.31 \%$ at E10.5) decrease (Fig. 1B). Next, we detected the chromosome-wide distribution of hypomethylated promoter in IVF embryos at E7.5 and E10.5 respectively. Our results indicated that hypomethylated promoters were globally distributed across all autosomes and sex chromosomes (Fig. 1C), and the number of hypomethylated promoters was considerably higher than that of hypermethylated promoters on each chromosome (Fig. 1D). Next, a Venn diagram showed that a substantial proportion of promoters that should gain DNA methylation (upregulated from E3.5 to E7.5 with FC > 1.5) during implantation were hypomethylated in IVF E7.5 embryos (Fig. 1E). Phenotype annotations from
Mouse Genome Informatics (MGI) database further demonstrated that majority of those genes were functionally involved in embryonic developmental and survival, as well as postnatal growth. Collectively, these results indicate that IVF embryos may undergo impaired de novo DNA methylation during implantation, and thus leading to global hypomethylation by postimplantation stage.

\section{Impaired de novo DNA methylation transcriptionally participate in processes essential for embryonic development and survival}

Given DNA methylation at postimplantation stage plays an essential role in transcriptional regulation of developmental genes [26, 39], we next attempted to test the developmental consequence of impaired de novo DNA methylation. To this end, we performed an integrated analysis using our previously published MeDIP-seq data and transcriptome data that profiles DNA methylation and gene expression maps of IVO and IVF postimplantation embryos, respectively [31, 35]. There were 288 and 403 putative genes that were dysregulated due to impaired de novo DNA methylation at E7.5 and E10.5 respectively (Fig. 2A and D). GO and KEGG analyses indicated that those genes participated in many basic processes and pathways, such as endocytosis, cytolysis, cell differentiation, Notch and MAPK signaling pathways, as well as cellular response to stress stimulus (Fig. 2B, C, E, F). Moreover, based on MGI database, we found that genes enriched in KEGG analysis, were tightly associated with embryonic development and survival throughout the pregnancy (Fig. 2G and H).

\section{Dnmt3b is consistently inhibited during the transition from the blastocyst to post- implantation stage in IVF embryos}

Given the DNMT3B is the main enzyme required for de novo DNA methylation during early development $[26,28]$, we next attempted to test if Dnmt3b was inhibited in IVF embryos during early development. Analysis of dynamic transcriptome of IVO and IVF embryos revealed that Dnmt3b was consistently inhibited during the transition from the blastocyst to postimplantation stage (Fig. 3A). In addition, the inhibited Dnmt3b expression was further confirmed on the protein level in the blastocyst (Fig. 3B) and E7.5 embryos (Fig. 3C). Those results demonstrate that IVF processes induce a significant decrease in Dnmt3b/DNMT3B in early embryos, which may be the potential target for correcting DNA methylation defects in IVF postimplantation embryos. 


\section{Repression of FGF signaling is responsible for inhibited Dnmt3b expression in IVF pre- implantation embryos}

Having confirmed the consistent repression of Dnmt3b/DNMT3B in early IVF embryos, we next attempted to identify the critical reasons that contributed to Dnmt3b inhibition in IVF embryos. Given the transcriptome data suggested Dnmt3b was transcriptionally inhibited as early as the preimplantation stage (Fig. 3A) we first confirmed this inhibition in IVF blastocysts using RT-qPCR analysis (Fig. 4A). In addition, by reanalyzing previously published [40] and our own transcriptome data, we found medium components (Fig. 4B), but not oxygen concentration of culture conditions (Fig. 4C), can increase Dnmt3b expression.
A

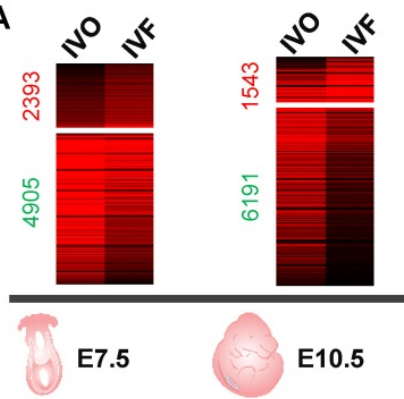

C

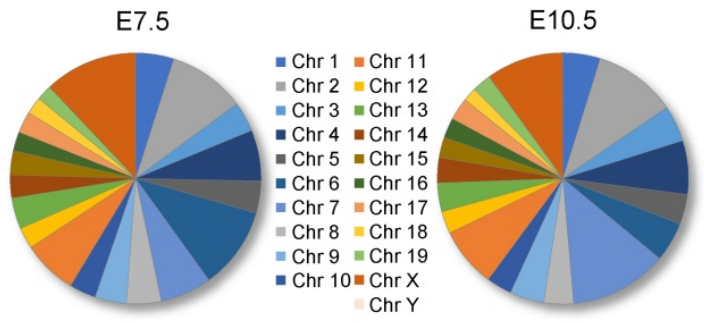

D

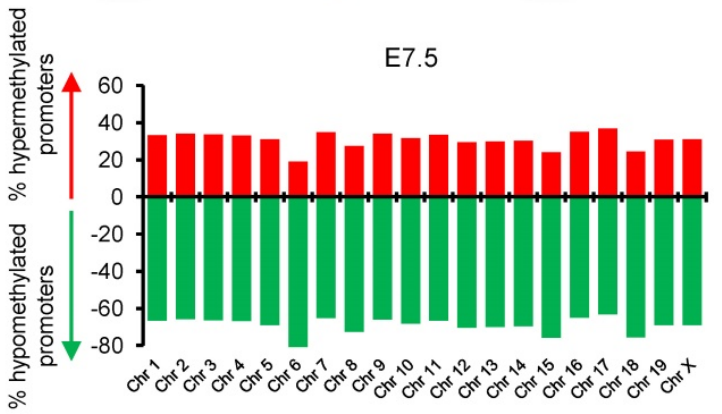

B

No. of hypermethylated $(\mathrm{FC}>1.5)$ promoters in IVF embryos

No. of hypomethylated $(\mathrm{FC}>1.5)$ promoters in IVF embryos

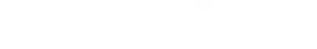

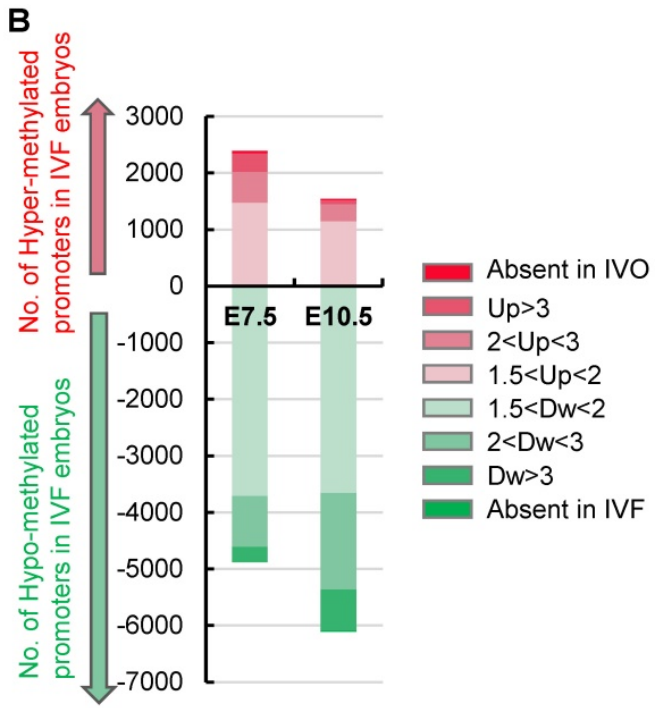

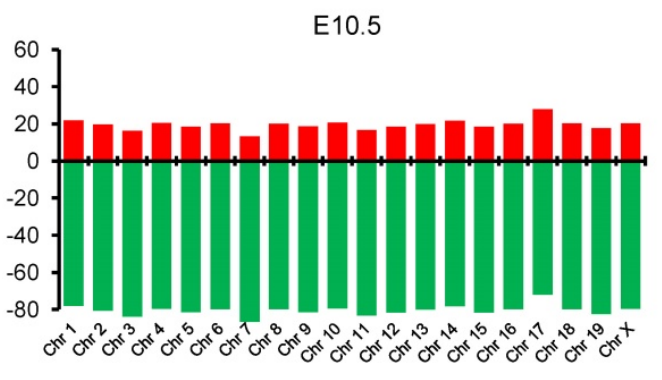

E

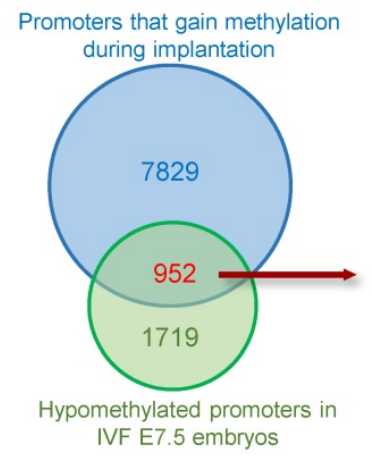

\begin{tabular}{|c|l|}
\hline Gene & \multicolumn{1}{|c|}{ MGI phenotype } \\
\hline Dpm3 & Preweaning lethality, embryonic lethality prior to organogenesis \\
\hline G6pdx & Embryonic growth arrest, embryonic lethality during organogenesis \\
\hline Ogt & Prenatal lethality, decreased fetal size, female infertility \\
\hline Hormad1 & Embryonic growth arrest, failure of embryo implantation, infertility \\
\hline Rp/13a & Premature death, abnormal lung morphology \\
\hline Ppp3r1 & Embryonic lethality during organogenesis, premature death \\
\hline Lef1 & Perinatal lethality, complete penetrance \\
\hline Gphn & Neonatal lethality, complete penetrance \\
\hline Hoxa13 & Lethality throughout fetal growth and development, complete penetrance \\
\hline Bsn & Premature death \\
\hline
\end{tabular}

Figure 1. IVF embryos exhibit global hypomethylation by postimplantation stage. (A) Comparations of promoter DNA methylation in IVF or IVO embryos at E7.5 or E10.5. Red number and green numbers indicate hypermethylated (FC > 1.5) or hypomethylated (FC > 1.5) promoters in IVF embryos, respectively. Total data are shown in Table S5 and S6. 822 IVO and 795 IVF embryos at E7.5, 324 IVO and 278 IVF embryos at E10.5 were respectively used in this experiment. (B) Contribution of promoters with different fold changes in IVF embryos. (C) The chromosome-wide distribution of hypomethylated promoters in IVF embryos at E7.5 and E10.5 respectively. (D) Proportions of hypermethylated and hypomethylated promoters on each chromosome. (E) The Venn diagram of genes that should gain promoter methylation during implantation stage (FC E7.5/E3.5 > 1.5) and hypomethylated promoters in IVF E7.5 embryos (FC E7.5 IVF/IVO < 0.67). MGI phenotypes of overlapped genes are showed in right panel (top 10) and Table S2. 

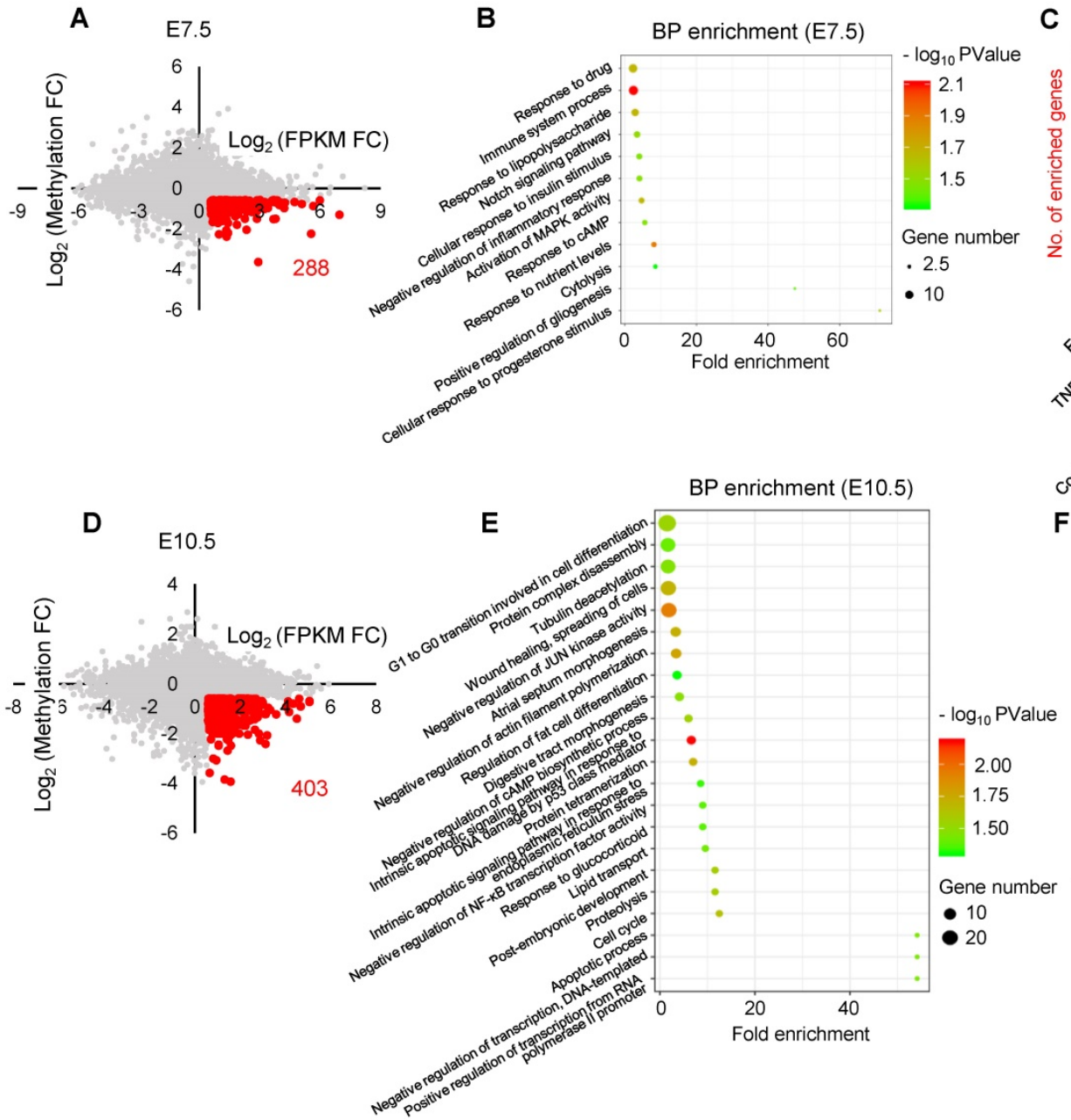

C KEGG_pathway (E7.5)

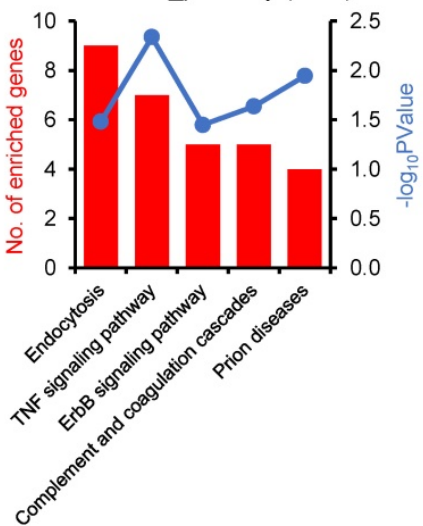

$\mathbf{F}$ KEGG_pathway (E10.5)

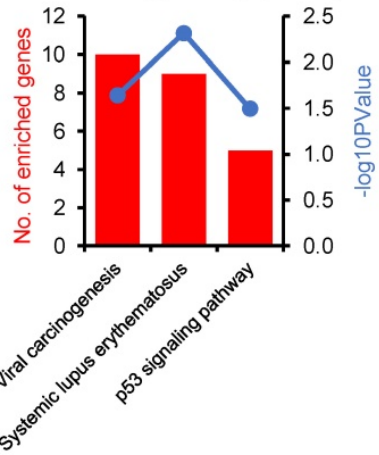

G

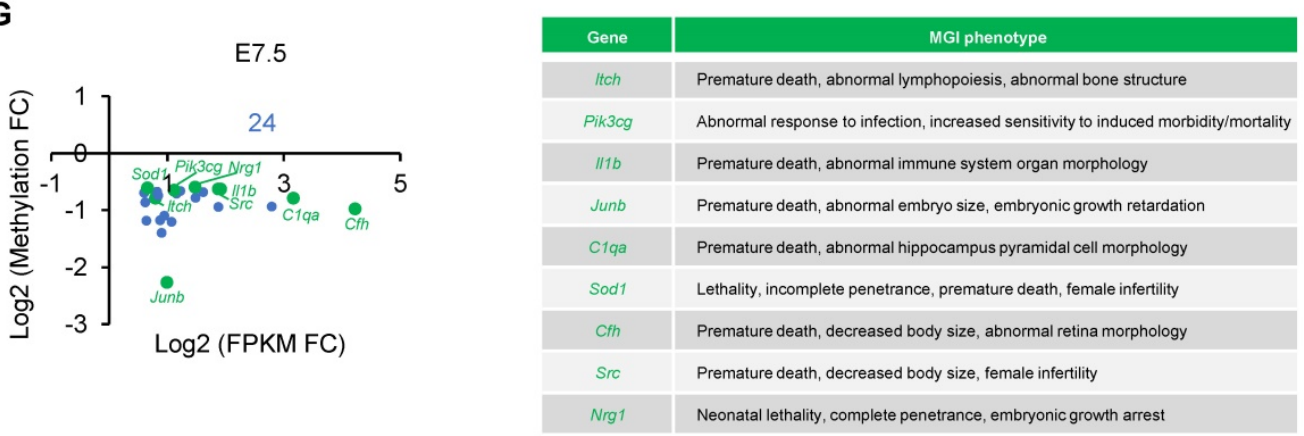

H

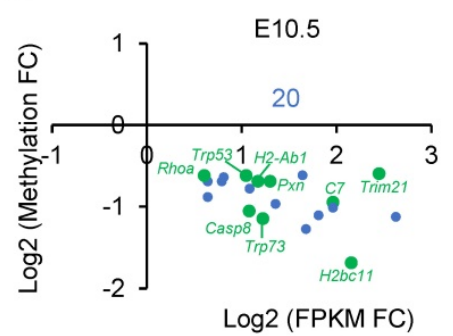

\begin{tabular}{|c|l|}
\hline Gene & \multicolumn{1}{|c|}{ MGI phenotype } \\
\hline$C 7$ & Premature death, increased neuron apoptosis, neurodegeneration \\
\hline$H 2-A b 1$ & Premature death, postnatal growth retardation, \\
\hline$P \times n$ & Decreased embryo size, embryonic lethality during organogenesis \\
\hline$T r 53$ & Lethality throughout fetal growth and development, complete penetrance \\
\hline Casp8 & Embryonic lethality during organogenesis, complete penetrance \\
\hline$R$ Rho & Embryonic lethality, complete penetrance, abnormal neuron proliferation \\
\hline$T r p 73$ & Premature death, abnormal embryo development \\
\hline H2bc11 & Abnormal olfactory sensory neuron morphology, impaired olfaction \\
\hline$T r i m 21$ & Abnormal immune system morphology, abnormal interleukin level \\
\hline
\end{tabular}

Figure 2. Functional analysis of dysregulated genes due to hypomethylated promoters in IVF postimplantation embryos. (A and D) $A$ scatter plots of putative genes (red dots, $\log _{2}$ FPKM FC > 0.58) that are dysregulated due to impaired de novo DNA methylation (Log 2 Methylation FC $<-0.58$ ) in IVF embryos at E7.5(A) or E10.5 (D), respectively. (B and E) Biological process (BP) enrichment based on functional annotation of GO terms using filtered genes at E7.5 (B) or E10.5 (E). (C and F) KEGG analysis of genes enriched by BP enrichment at E7.5 (C) or E10.5 (F). The left ordinate indicates the number of enriched genes in each term, and the right ordinate indicates enrichment sore. $(\mathrm{G}$ and $\mathrm{H})$ The location of genes enriched by KEGG analysis at E7.5 $(\mathrm{G})$ or E10.5 $(\mathrm{H})$. Genes that have MGI phenotypes related to embryos development are highlighted as green dots. Complete MGI phenotypes are showed in Table S3 and Table S4. 
A
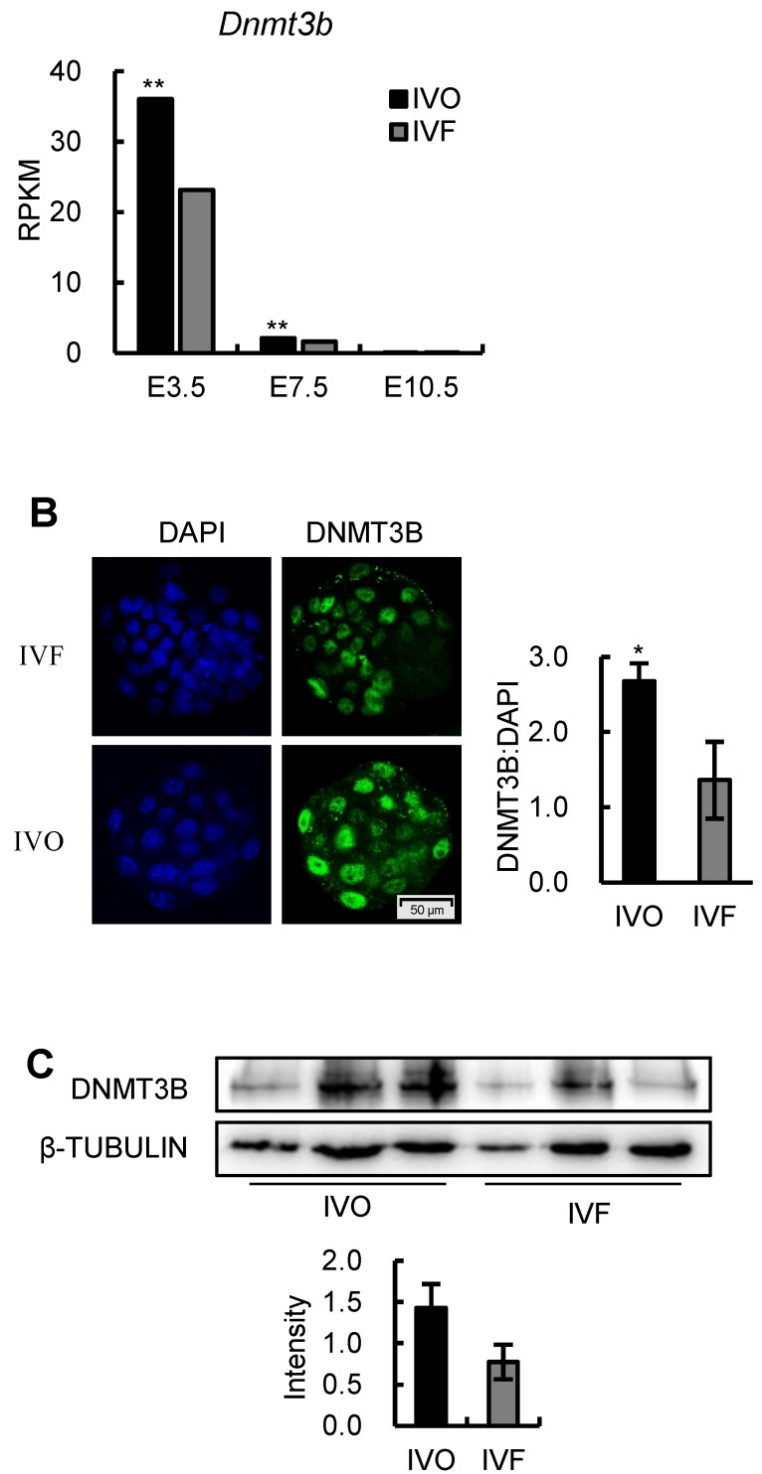

Figure 3. Dnmt3b is consistently inhibited during the transition from the blastocyst to postimplantation stage in IVF embryos. (A) Comparison of expression levels of Dnmt3b between IVO or IVF embryos at E3.5, E7.5 or E10.5 using transcriptome data. (B) Immunofluorescence staining of DNMT3B in IVO (24 embryos) or IVF (21 embryos) blastocysts and relative quantification of DNMT3B signals (right panel). (C) Western blot analysis of DNMT3B in IVO or IVF E7.5 embryos and relative quantification of DNMT3B levels (lower panel). Three pools were used to perform WB in IVO or IVF group, with 3 embryos in each pool. $* P<$ $0.05, * * P<0.01$

Since previous studies demonstrated that FGF signaling plays a critical role in stimulating Dnmt3b expression [41-43], we next profiled expression patterns of FGF family members in early embryos and maternal oviduct, using our transcriptome data. We found many FGF ligands that are tightly associated with embryonic development and survival was repressed in IVF embryos (Fig. 4D and E). In addition, we noticed that FGF2, which can substitute for FGF4 to rescue the developmental defects in FGF4-deficient mouse embryos [44], was exclusively produced maternal oviduct (Fig. 4F). This observation, together with fact that oviduct fluid supplementation can stimulate Dnmt3b expression (Fig. 4B), led us to ask if FGF2 supplementation can rescue $D n m t 3 b$ inhibition in IVF embryos. To test this, standard in vitro culture medium was supplemented with FGF2 at different concentrations. As expected, FGF2 supplementation significantly increased the expression levels of Dnmt3b, as well as Dnmt3a and Dnmt3l (Fig. 4G), and thus enhanced DNA methylation levels in IVF embryos (Fig. $4 \mathrm{H}$ ). In addition, immunofluorescence analysis of CDX2 and EdU staining indicated that FGF2 also facilitate trophectoderm (TE) differentiation (Fig. 4I) and proliferation (Fig. 4J) during blastocyst formation. Finally, morphological quantifications also showed the beneficial effect of FGF2 supplementation in accelerating developmental progression in IVF embryos (Fig. 4K). Collectively, these results suggest that repression of FGF signaling, especially the deficiency of paracrine FGF2 from oviduct during preimplantation stage, may be the main cause of Dnmt3b inhibition in IVF blastocysts.

\section{FGF signaling remains repressed in IVF post- implantation embryos and induces DNA hypo- methylation during early development}

Having established a correlation between repression of FGF signaling and Dnmt3b inhibition in preimplantation embryos, we next attempted to address whether FGF signaling remains repressed in IVF embryos following transfer to recipients. The remarkable morphological abnormalities of IVF embryos were observed as early as the stage shortly after implantation (Fig. 5A), which is similar with that reported our previous study [13]. Given that FGF4 is a key factor required for embryonic development following blastocyst stage and essential for successful implantation [44-46], we next compared Fgf4 expression between IVO and IVF postimplantation embryos using our transcriptome data. We found $F g f 4$ remained repressed in IVF E7.5 embryos (Fig. 5B), which was positively correlated with the Dnmt3b inhibition at this stage (Fig. 5C).

Next, we attempted to determine if consistent repression of FGF signaling is responsible for Dnmt3b inhibition and DNA hypomethylation in IVF postimplantation stage. Because in vivo intervention of embryonic FGF signaling is technically inefficient, we used mouse ES cell differentiation as a model, which recapitulated the process of de novo DNA methylation during implantation $[47,48]$. It should be mentioned that ES cell differentiation was induced in serum-free medium, to exclude the possible influence of serum FGF ligand. CRISPR/CAS9n-based genome editing system was used to knockout Fgf4 gene in mESCs (Fig. 5D). Comparing with wild type cells, the 
expression levels of Dnmt3b gene in Fgf4-knockout (Fgf4-KO) ES cells were consistently inhibited during differentiation, which can be completely restored by exogenous supplementation of FGF4 (Fig. 5E). These findings were also confirmed on protein levels (Fig.
$5 \mathrm{~F})$. Correspondingly, results of $5-\mathrm{mC}$ staining of 5 days differentiated Fgf4-KO ES cells showed a remarkable DNA hypomethylation (Fig. 5G), suggesting that repression of FGF signaling impairs the process of de novo DNA methylation.
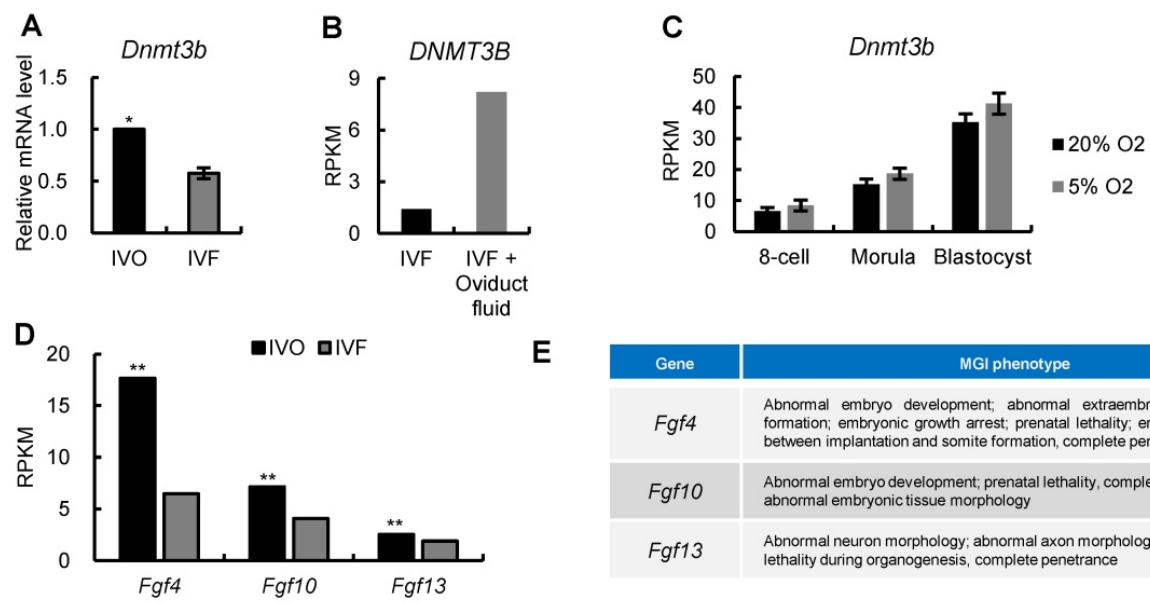

E

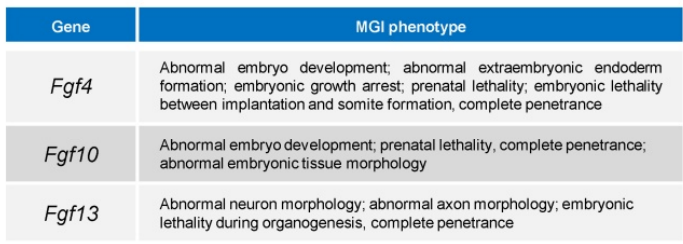

F

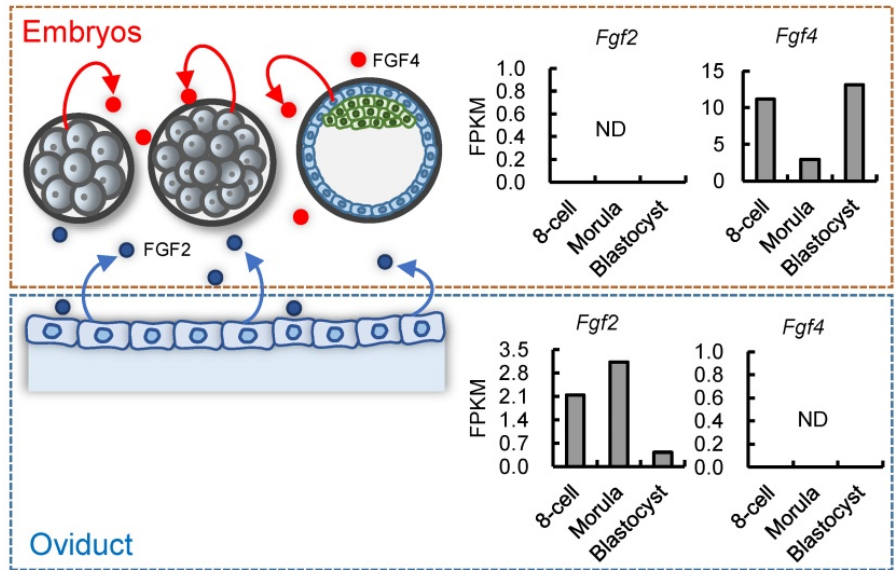

G

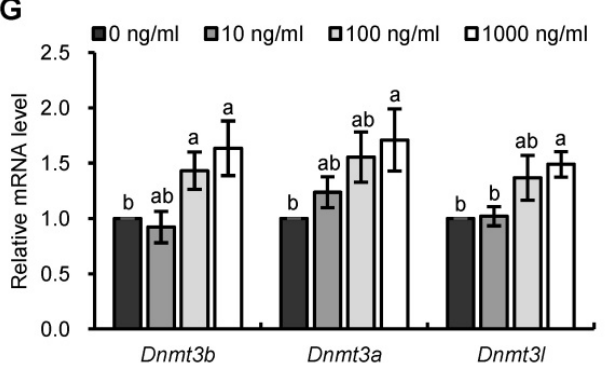

K

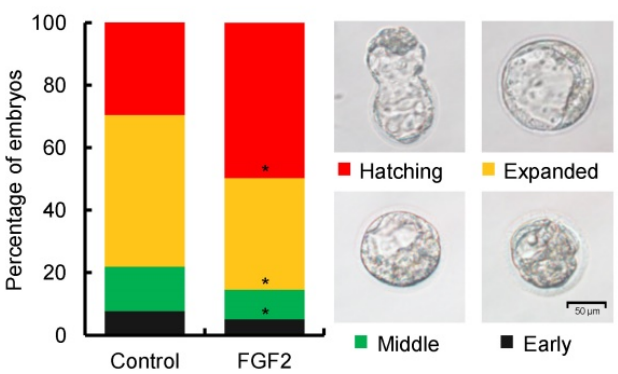

H
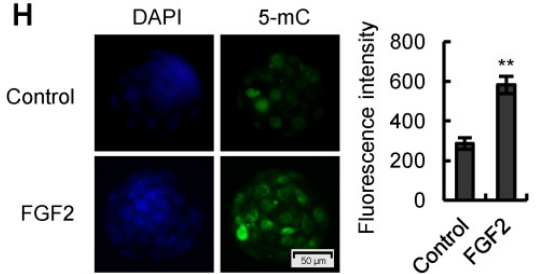

I

DAPI
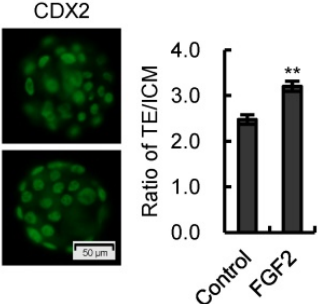

$J$

DAPI

\section{EdU}

Control

FGF2

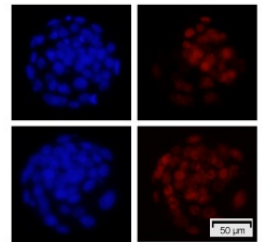

Figure 4. Repression of FGF signaling is responsible for inhibited Dnmt3b expression in IVF preimplantation embryos. (A) Relative mRNA expression levels of Dnmt3b in IVO and IVF blastocysts using RT-qPCR analysis. Experiments were replicated three times. At least 25 embryos were collected in each group. (B) Comparison of 
DNMT3B expression levels in IVF and IVF + oviduct fluid porcine embryos using previously published transcriptome data [40]. (C) Dynamic comparisons of Dnmt3b expression levels using transcriptome data of IVF mouse preimplantation embryos under $20 \%$ or $5 \% \mathrm{O}_{2}$ condition. (D) Comparison of Fgf4, FgflO and Fgfl 3 expression levels in IVO and IVF blastocysts. (E) MGI phenotypes of main FGF members that are inhibited in IVF blastocysts. (F) Diagram of expression patterns of autocrine and paracrine FGF ligands during preimplantation stage. Right panel in each box shows expression levels of autocrine and paracrine FGF ligands in embryos themselves and the oviducts. (G) Expression levels of Dnmt3b, Dnmt3a and Dnmt3l in IVF blastocysts cultured in medium supplemented with $10 \mathrm{ng} / \mathrm{ml}, 100 \mathrm{ng} / \mathrm{ml}$ or $1000 \mathrm{ng} / \mathrm{ml} \mathrm{FGF2.} \mathrm{Experiments} \mathrm{were} \mathrm{replicated} \mathrm{four} \mathrm{times.} 30 \sim 56$ embryos were collected in each group. $(\mathrm{H})$ Immunofluorescence staining of 5-mC in IVF blastocysts cultured in control medium (33 embryos) or medium supplemented with $1000 \mathrm{ng} / \mathrm{ml} \mathrm{FGF2} \mathrm{(31} \mathrm{embryos).} \mathrm{Right} \mathrm{panel} \mathrm{shows} \mathrm{relative} \mathrm{fluorescence} \mathrm{intensity} \mathrm{of} \mathrm{5-mC} \mathrm{(5-mC/DAPI).} \mathrm{(I)} \mathrm{Immunofluorescence} \mathrm{staining} \mathrm{of} \mathrm{CDX2} \mathrm{in} \mathrm{IVF} \mathrm{blastocysts} \mathrm{treated} \mathrm{with}$ (44 embryos) or without (6 embryos) exogenous FGF2. Right panel shows ratio of TE/ICM in IVF blastocysts treated with or without exogenous FGF2. (J) EdU staining (left) and quantification of the ratio of EdU/DAPI (right) in IVF blastocysts treated with (39 embryos) or without (39 embryos) exogenous FGF2. (K) Percentage of embryos at different developmental stages in the culture medium with or without exogenous FGF2. Experiments were replicated five times. 39 69 embryos were collected in each group. Representative embryo pictures were showed in the right panel. Values with different letters are significantly different $(P<0.05)$. $* P<0.05$, $* * P<0.01$.

A IVO

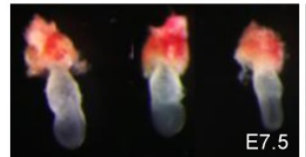

IVF

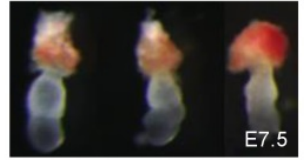

Normal

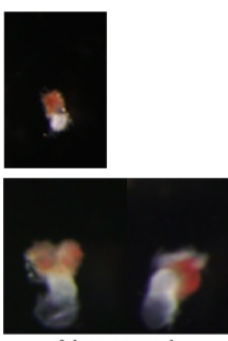

Abnormal

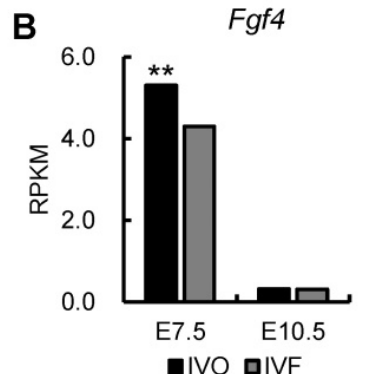

aIVO 口IVF

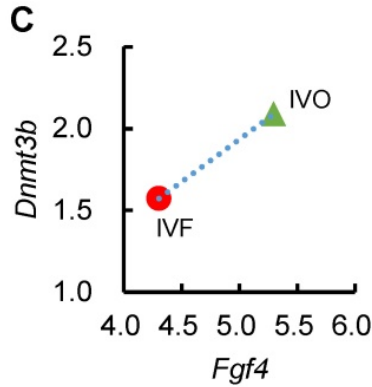

E

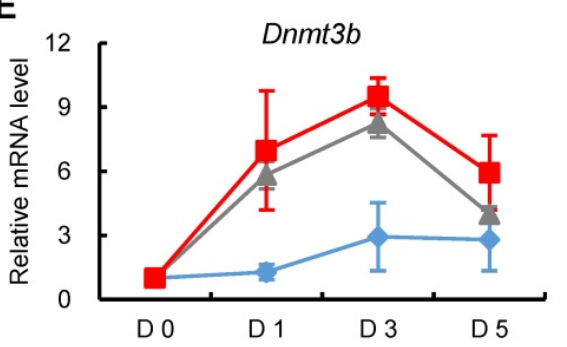

$\longrightarrow$ WT $\longrightarrow$ Fgf4-KO $\longrightarrow$ Fgf4-KO + FGF4

Day 5 of differentiation

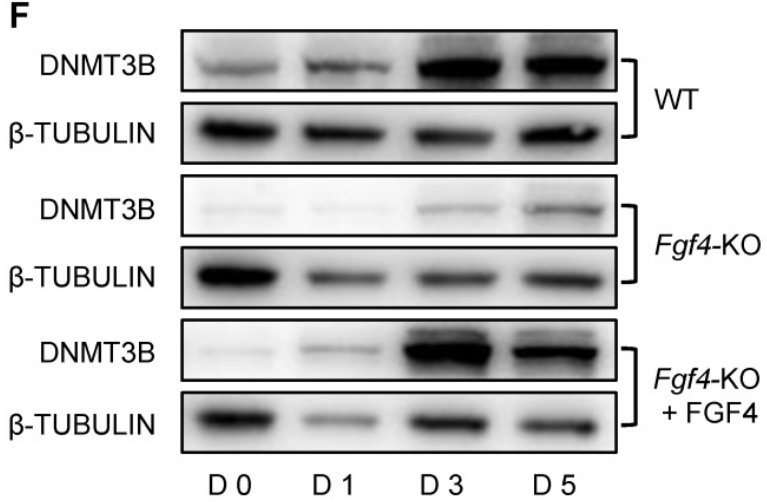

$\mathbf{G}$

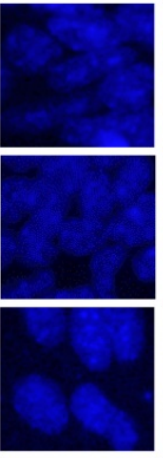

DAPI

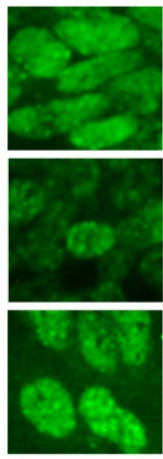

5-mC

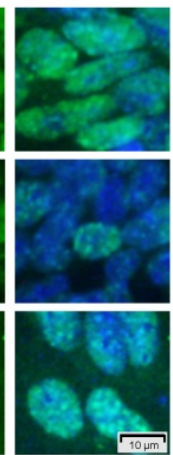

MERGE

Figure 5. FGF signaling remains repressed in IVF postimplantation embryos and induces DNA hypomethylation during early development. (A) Representative images of morphologically normal and abnormal IVO or IVF embryos at E7.5. (B) Comparison of expression levels of Fgf4 between IVO or IVF embryos at E7.5 or E10.5. (C) The positive correlation between Fgf4 and Dnmt3b expression levels in IVO and IVF E7.5 embryos. (D) Flow diagram of CRISPR/CAS9n-based knockout of Fgf4. (E and F) The relative mRNA (E) or protein (F) levels of Dnmt3b/DNMT3B in WT, Fgf4-KO, or Fgf4-KO+FGF4 ES cells on Day 0, 1, 3 and 5 of differentiation. Experiments were triple replicated, and representative WB images were exhibited. (G) Immunofluorescence staining of 5-mC in WT, Fgf4-KO or Fgf4-KO + FGF4 on Day 5 of differentiation. ** $P<0.01$.

\section{FGF signaling regulates Dnmt3b expression in early embryos through MEK/ERK-SPI pathway}

To further understand the mechanism underlying Dnmt3b inhibition and impaired de novo DNA methylation which could be used as the potential target for correcting developmental defects of IVF embryos, we next tested the involvement of ERK signaling, the primary downstream pathway of FGF signaling, in mediating FGF-induced Dnmt3b expression. The beneficial effect of FGF2 supplementation on increasing Dnmt3b expression in IVF blastocyst, could be completely attenuated by supplementing MEK/ERK inhibitor PD0325901 (PD) (Fig. 6A). This result was further confirmed on protein levels using immunofluorescence staining of 
DNMT3B (Fig. 6B and C). Correspondingly, both mRNA and protein analyses from ES cells showed that Dnmt3b/DNMT3B expression were completely absent when FGFR and MEK/ERK pathway were blocked by inhibitors respectively (Fig. 6D and E). In addition, we also found either FGFR or MEK/ERK inhibition led to DNA hypomethylation in 5 days differentiated ES cells (Fig. 6F). Those results demonstrated that FGF signaling regulates Dnmt3b expression and DNA methylation levels during early development through FGFR-MEK/ERK pathway.

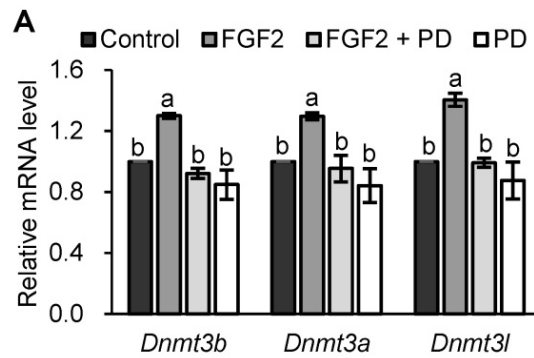

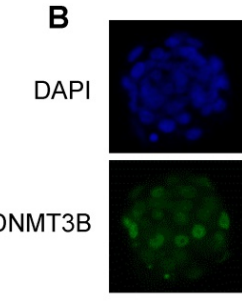

Control

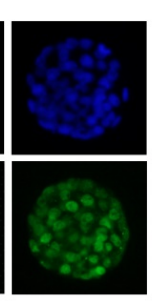

FGF2

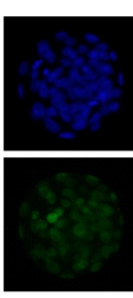

$F G F 2+P D$

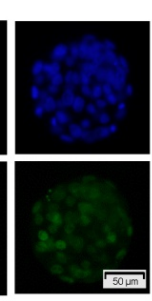

PD

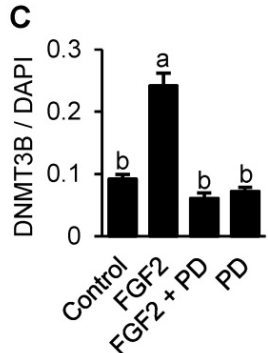

E

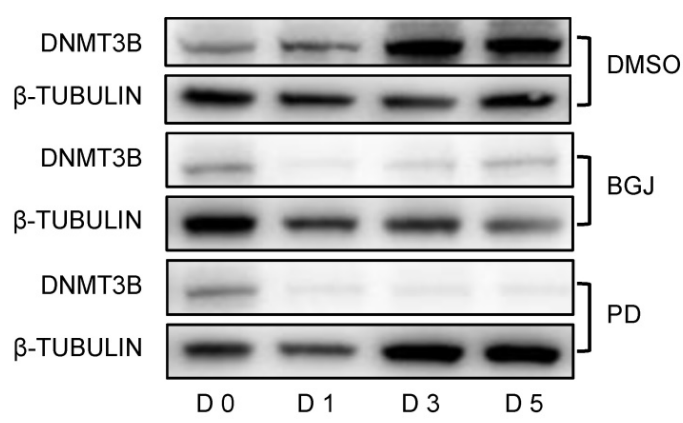

G

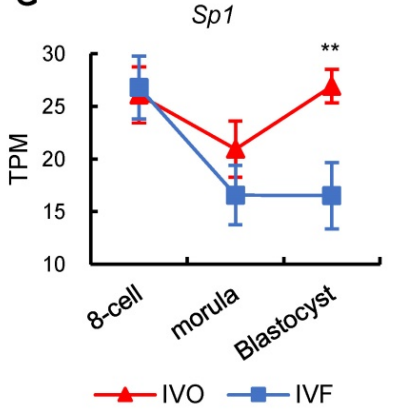

H

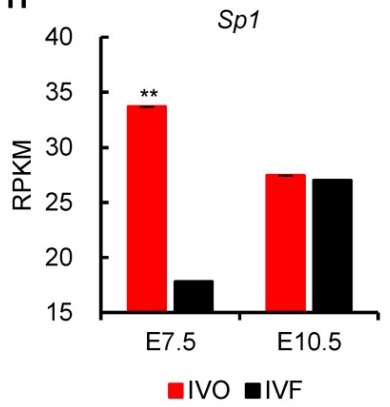

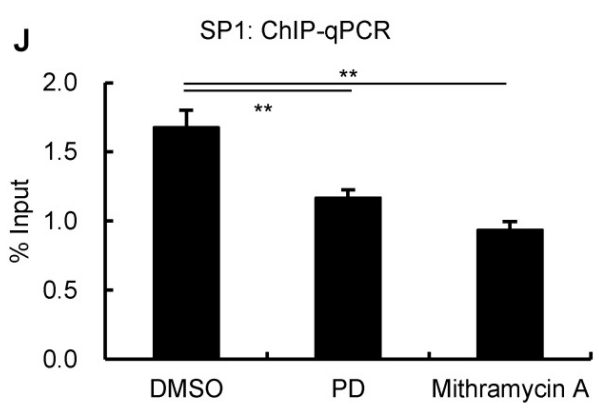

Figure 6. FGF signaling regulates Dnmt3b expression early development through MEK/ERK-SP1 pathway. (A) Relative expression levels of Dnmt3b, Dnmt3a and Dnmt3l in IVF blastocysts treated with FGF2, FGF2+PD (MEK/ERK inhibitor) and PD respectively. Experiments were replicated three times. 18 43 embryos were collected in each group. (B and C) Immunofluorescence staining (B) and quantification (C) of DNMT3B in IVF blastocysts treated with FGF2 (42 embryos), FGF2+PD (30 embryos) and PD (30 embryos) respectively. 63 embryos were used as control. (D and E) Relative mRNA (D) and protein (E) levels of Dnmt3b/DNMT3B in ES cells treated with DMSO, BGJ (nonspecific FGFR inhibitor) and PD on Day 0, 1, 3 and 5 of differentiation. Experiments were triple replicated, and representative WB images were exhibited. (F) Immunofluorescence staining of 5-mC in ES cells treated with DMSO, BGJ and PD on Day 5 of differentiation. (G and H) Dynamic comparisons of expression levels of SpI in IVO or IVF embryos during preimplantation (G) and postimplantation (H) stage using transcriptome data. (I) Dynamic comparisons of expression levels of SpI in WT or Fgf4-KO ES cells on Day $0,1,3$ and 5 of differentiation. Experiments were triple replicated. (J) ChIP analysis of SPI enrichment at a distal binding site that is located $10 \mathrm{~kb}$ upstream of Dnmt3b in ES cells treated with DMSO, PD and Mithramycin A (nonspecific SPI inhibitor). ChIP-qPCR were repeated for three times. Values with different letters are significantly different $(P<0.05)$. $* P<0.05, * * P<0.01$. 
Because results from previous study showed that a general transcription factor specificity protein 1 (SP1) participates in the transcriptional regulation Dnm3b [49], we next test the involvement of SP1 in Dnmt3b inhibition. Results from transcriptome data showed that Sp1 was consistently inhibited in IVF embryos from preimplantation to postimplantation stage (Fig. 6G and H), which coincides with the FGF signaling and Dnmt3b inhibition. More importantly, using Fgf4-KO ES cells, we found transcriptional upregulation of $S p 1$ was also largely dependent on FGF4 during ES differentiation (Fig. 6I), indicating that SP1 may play an important role in mediating FGF-MEK/ERK-induced Dnmt3b expression during early development. To provide more direct evidence supporting the mediating role of SP1 in regulating Dnmt3b expression, we screened Dnmt3b promoter regions and SP1-ChIP data in Cistrome Data Browser. Results revealed several potential SP1 binding sites in Dnmt3b promoter regions. Results from highsensitivity immunoprecipitation showed that SP1 are significantly enriched in Dnmt3b promoter regions, but the binding could be significantly attenuated by blocking MEK/ERK pathway, or supplementing mithramycin A, a non-specific inhibitor that displaces transcriptional activators which bind to GC-rich regions of promoters such as SP1 (Fig. 6J). These results demonstrated that FGF signaling-induced Dnmt3b expression during early development is largely dependent on MEK/ERK-SP1 pathway.

\section{Discussion}

It has been widely accepted that a wave of genome-wide de novo DNA methylation that occurs around implantation period, re-establishes the DNA methylation patterns from a globally hypomethylated state in blastocysts to relatively static DNA methylation patterns in postimplantation embryos [26, 27, 50]. Focusing on promoters, which are main targets for transcriptional regulation of developmental genes in determining pluripotent or differentiated states [37, 38], and are preferentially de novo methylated during early development [26, 39], we find IVF embryos undergo an impaired de novo DNA methylation. According to previous studies using Dnmts-deficient mouse models, defects in de novo DNA methylation result in severe embryonic or postnatal lethality [28, 51], as well as aberrant organogenesis [52, 53]. This is in line with the morphological observation in our previous studies $[13,31]$, as well as the integrated bioinformatic analysis in the present study: many putative genes that were dysregulated due to impaired de novo DNA methylation participated in many basic processes and pathways (e.g., cell differentiation, cellular response to stress stimulus, Notch and MAPK signaling pathways, etc.) and were tightly associated with embryonic development and survival throughout the pregnancy.

DNA methyltransferase DNMT3B is the main enzyme required for de novo DNA methylation during early development. The genetic depletion of Dnmt3b leads to severe hypomethylation at pluripotency genes, gastrulation genes, germline-specific genes, hematopoietic genes, etc. [26, 39]. In addition, Dnmt3b-deficient mice show multiple developmental defects shortly after implantation and postimplantation embryonic lethality [28, 54]. In the present study, the observation of consistent Dnmt3b inhibition in IVF embryos, together with results of our own and other colleagues' studies [55, 56], suggest that the expression of methyltransferase is highly sensitive to perturbations of developmental environment. Correspondingly, we observed a global hypomethylation in IVF postimplantation embryos, indicating that IVF embryos undergo an impaired de novo DNA methylation during implantation stage. These results are in line with previous observations of genomic hypomethylation or global imprinting loss in IVF-conceived embryos, placentas, and offspring [17, 19, 57], since Dnmt3b was also thought to be involved in the maintenance of DNA methylation imprints during early development [28].

Our study further indicates that repression of FGF signaling is the main cause of Dnmt3b inhibition in IVF embryos during the transition from the blastocyst to postimplantation stage. Indeed, the critical mechanism responsible for initiation and maintenance of Dnmt3b expression during early development remains largely unknown. Although a previous study suggested that FGF signaling inhibition in ES cells induced repression of de novo methyltransferases, as well as global hypomethylation, via chemical-induced ERK1/2 and GSK3B signaling inhibition (2i) [41], the precise upstream signaling responsible for transcriptional activation of Dnmt3b remains elusive. Our study, using blastocysts and ES cells as models, indicates that FGF signaling is critical for initiation and maintenance of Dnmt3b expression. FGF signaling in the blastocysts is connection with the exit from pluripotency to differentiation. In particular, FGF4, which is the only FGF ligand produced by ICM cells in the blastocysts, is indispensable for lineage differentiation of pluripotent epiblast (Epi) and the primitive endoderm (PrE), the second fate decision of early embryos, and thus is required for postimplantation development $[44,46,58,59]$. The coincidence between this process and de novo DNA methylation raises the possibility that FGF signaling is 
also essential for stimulating Dnmt3b expression during early development. Evidences from Fgf4deficient ES cells support this hypothesis: Dnmt3b expression fails to be imitated and remains at low levels throughout differentiation, and this failure can be completely rescued by exogenous supplementation of FGF4. Of note, MEK/ERK-SP1 pathway plays a critical mediating role in this process. Considering that activation of MEK/ERK signaling mediates the effect of growth factors and is essential for early development and differentiation [60-62], MEK/ERKSP1 pathway may be a potential target for rescuing developmental defects of IVF embryos.

In addition to consistently inhibited Fgf4 expression in IVF embryos during the transition from the blastocyst to postimplantation stage, we also noticed that the absence of paracrine FGF signaling may also participate in Dnmt3b inhibition. FGF2 is a representative paracrine factor that is exclusively produced by the oviduct, but not by embryos during early development. Our results indicate that supplementation of FGF2, which is not present in any commercially available culture media, can restore Dnmt3b expression to the level comparable to that of IVO embryos, and this beneficial effect can be blocked by inhibiting MEK/ERK pathway. It should also be

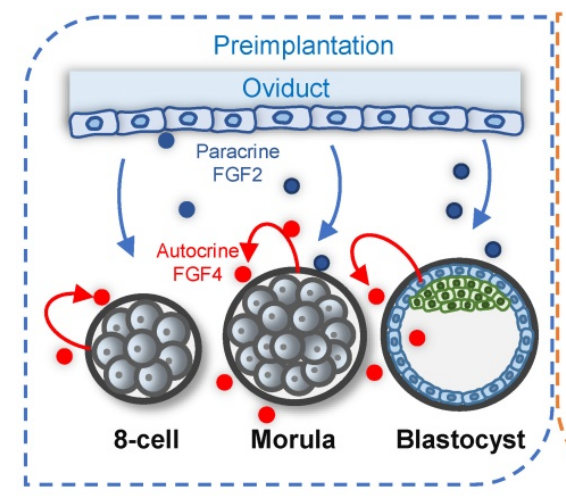

IVO embryos

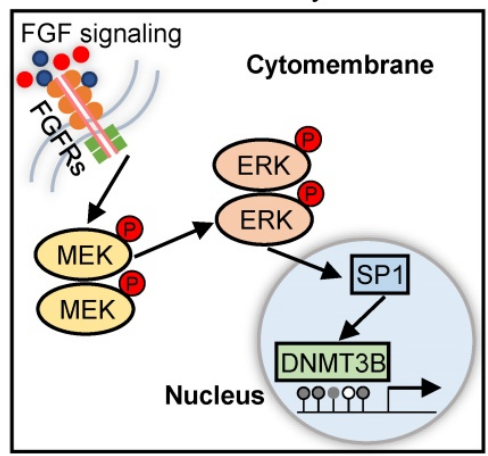

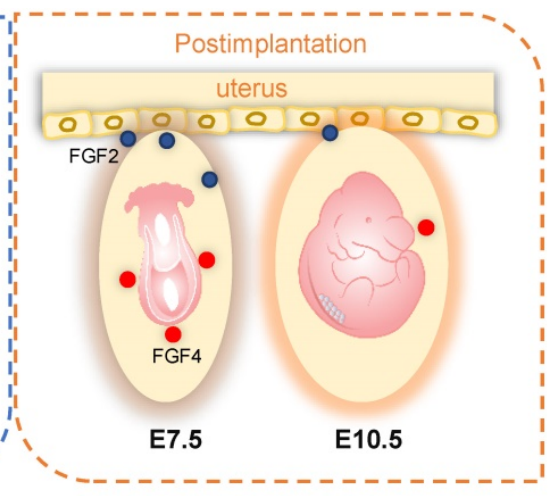

IVF embryos

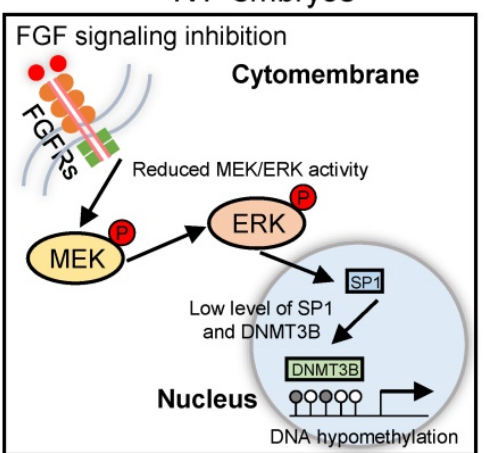

Figure 7. Schematic diagram illustrating the role of repression of FGF signaling in inducing Dnmt3b inhibition during early development of IVF embryos. Upper panels: early embryonic development during preimplantation stage are supported via the synergic effect of autocrine and paracrine FGF signaling. Blue and red dots represent FGF2 and FGF4 ligands, which are produced by the oviduct and embryos themselves, respectively. Lower panel: repression of FGF signaling underlies the mechanism responsible for Dnmt3b inhibition and hypomethylation in IVF embryos, MEK/ERK-SPI pathway plays an essential mediating role during this process. mentioned that in addition to upregulated $D n m+3 b$ expression and increased DNA methylation levels, FGF2 supplementation also changed lineage differentiation and proliferation of blastocysts. These observations, further emphasized the importance of DNA homeostasis in finetuning preimplantation development, as proposed by previous studies [41, 63, 64]. with the results reported by $\mathrm{P}$ Coy et al: DNA thylation and gene expression of IVF embryos can partially corrected in the presence of oviductal [40, 65]. Our results, together with results ported by $\mathrm{P}$ Coy et al., support the concept that maintenance of epigenetic features early embryos may be achieved via the synergic of autocrine and paracrine factors, which are embryos themselves and the oviduct, ectively. Given safety concerns of transmission of ove have fully been addressed after addition oviductal fluids in the culture media, it only been onimal reproduction at present, but not use. By contrast, the chemically defined medium that enhances developmental potential of IVF embryos is more reasonable, especially in the context of clinical practice of human assisted reproductive technologies. Until now, however, only very limited growth factors or cytokines that present in oviductal fluid are proven to be used in commercially available culture media [66]. Thus, identifying the developmental role of oviductal growth factors or cytokines in supporting early embryos, and thus formulating the culture media, may be a promising strategy.

Collectively, focusing on DNA hypomethylation in IVF postimplantation embryos, our study identifies that FGF signaling is essential for supporting Dnmt3b expression during early development, via the synergic effect of autocrine and paracrine FGF ligands. During in vitro development, repression of FGF signaling is responsible for consistent inhibition of Dnmt3b, the main de novo methyltransferase (Fig. 7). Our current finding not only reveals the main mechanism underlying DNA methylation defects in IVF embryos, but also 
proposes a potential strategy for preventing or correcting IVF-associated epigenetic errors via the use of oviductal growth factors or cytokines that can support early embryonic development.

\section{Supplementary Material}

Supplementary figures and tables.

http://www.ijbs.com/v16p3085s1.xlsx

\section{Acknowledgments}

PGK12.1 was a kindly gift from Neil Brockdorff at University of Oxford. This work was supported by grants from the National Key R\&D Program (2017YFD0501901 and 2017YFD0501905), and the National Natural Science Foundation of China (No. 3167246 and 31472092).

\section{Competing Interests}

The authors have declared that no competing interest exists.

\section{References}

1. [Internet] ESHRE (2020) ART fact sheet. https://www.eshre.eu/-/media/ sitecore-files/Press-room/ART-fact-sheet-2020-data-2016.pdf

2. Bergh T, Ericson A, Hillensjö T, et al. Deliveries and children born after in-vitro fertilisation in Sweden 1982-95: a retrospective cohort study. Lancet. 1999; 354(9190): 1579-85.

3. Racowsky C. High rates of embryonic loss, yet high incidence of multiple births in human ART: is this paradoxical? Theriogenology. 2002; 57(1): 87-96.

4. Schieve LA, Meikle SF, Ferre C, et al. Low and very low birth weight in infants conceived with use of assisted reproductive technology. N Engl J Med. 2002; 346(10): 731-7.

5. Klemetti R, Sevón T, Gissler M, et al. Health of children born as a result of in vitro fertilization. Pediatrics. 2006; 118(5): 1819-27.

6. Reefhuis J, Honein MA, Schieve LA, et al. Assisted reproductive technology and major structural birth defects in the United States. Hum Reprod. 2009; 24(2): 360-6.

7. Hart R, Norman RJ. The longer-term health outcomes for children born as a result of IVF treatment: Part I--General health outcomes. Hum Reprod Update. 2013; 19(3): 232-43.

8. Källén $\mathrm{B}$, Finnström $\mathrm{O}$, Lindam $\mathrm{A}$, et al. Cancer risk in children and young adults conceived by in vitro fertilization. Pediatrics. 2010; 126(2): 270-6.

9. Ceelen M, Weissenbruch MMv, Vermeiden JPW, et al. Cardiometabolic differences in children born after in vitro fertilization: follow-up study. J Clin Endocrinol Metab. 2008; 93(5): 1682-8.

10. Servick K. Unsettled questions trail IVF's success. Science. 2014; 345(6198): 744-6.

11. Rexhaj E, Paoloni-Giacobino A, Rimoldi SF, et al. Mice generated by in vitro fertilization exhibit vascular dysfunction and shortened life span. J Clin Invest. 2013; 123(12): 5052-60.

12. Sutcliffe AG, Peters CJ, Bowdin S, et al. Assisted reproductive therapies and imprinting disorders - a preliminary British survey. Hum Reprod. 2006; 21(4): 1009-11.

13. Tan K, An L, Miao K, et al. Impaired imprinted X chromosome inactivation is responsible for the skewed sex ratio following in vitro fertilization. Proc Natl Acad Sci U S A. 2016; 113(12): 3197-202.

14. Halliday J, Oke K, Breheny S, et al. Beckwith-Wiedemann syndrome and IVF: a case-control study. Am J Hum Genet. 2004; 75(3): 526-8.

15. Johnson JP, Schoof J, Beischel L, et al. Detection of a case of Angelman syndrome caused by an imprinting error in 949 pregnancies analyzed for AS following IVF. J Assist Reprod Genet. 2018; 35(6): 981-4.

16. Maher ER, Brueton LA, Bowdin SC, et al. Beckwith-Wiedemann syndrome and assisted reproduction technology (ART). J Med Genet. 2003; 40(1): 62-4.

17. Katari S, Turan N, Bibikova M, et al. DNA methylation and gene expression differences in children conceived in vitro or in vivo. Hum Mol Genet. 2009; 18(20): 3769-78.

18. Vermeiden JPW, Bernardus RE. Are imprinting disorders more prevalent after human in vitro fertilization or intracytoplasmic sperm injection? Fertil Steril. 2013; 99(3): 642-51.

19. Chen Z, Hagen DE, Elsik CG, et al. Characterization of global loss of imprinting in fetal overgrowth syndrome induced by assisted reproduction. Proc Natl Acad Sci U S A. 2015; 112(15): 4618-23.
20. Chen Z, Robbins KM, Wells KD, et al. Large offspring syndrome: a bovine model for the human loss-of-imprinting overgrowth syndrome BeckwithWiedemann. Epigenetics. 2013; 8(6): 591-601.

21. Deshmukh RS, Østrup O, Østrup E, et al. DNA methylation in porcine preimplantation embryos developed in vivo and produced by in vitro fertilization, parthenogenetic activation and somatic cell nuclear transfer. Epigenetics. 2011; 6(2): 177-87.

22. Li T, Vu TH, Ulaner GA, et al. IVF results in de novo DNA methylation and histone methylation at an Igf2-H19 imprinting epigenetic switch. Mol Hum Reprod. 2005; 11(9): 631-40.

23. Li L, Wang L, Xu X, et al. Genome-wide DNA methylation patterns in IVFconceived mice and their progeny: a putative model for ART-conceived humans. Reprod Toxicol. 2011; 32(1): 98-105.

24. Rivera RM, Stein P, Weaver JR, et al. Manipulations of mouse embryos prior to implantation result in aberrant expression of imprinted genes on day 9.5 of development. Hum Mol Genet. 2008; 17(1): 1-14.

25. Wright K, Brown L, Brown G, et al. Microarray assessment of methylation in individual mouse blastocyst stage embryos shows that in vitro culture may have widespread genomic effects. Hum Reprod. 2011; 26(9): 2576-85.

26. Borgel J, Guibert S, Li Y, et al. Targets and dynamics of promoter DNA methylation during early mouse development. Nat Genet. 2010; 42(12): 1093-100.

27. Smith ZD, Chan MM, Mikkelsen TS, et al. A unique regulatory phase of DNA methylation in the early mammalian embryo. Nature. 2012; 484(7394): 339-44.

28. Okano M, Bell DW, Haber DA, et al. DNA methyltransferases Dnmt3a and Dnmt $3 \mathrm{~b}$ are essential for de novo methylation and mammalian development. Cell. 1999; 99(3): 247-57.

29. Nagy A, Gerstenstein M, Vintersten K, et al. Manipulating the Mouse Embryo - A Laboratory Manual. New York, USA: Cold Spring Harbor Press; 2003.

30. Tan K, Wang X, Zhang Z, et al. Downregulation of miR-199a-5p Disrupts the Developmental Potential of In vitro-Fertilized Mouse Blastocysts. Biol Reprod. 2016; 95(3): 54

31. Ren L, Wang Z, An L, et al. Dynamic comparisons of high-resolution expression profiles highlighting mitochondria-related genes between in vivo and in vitro fertilized early mouse embryos. Hum Reprod. 2015; 30(12): 2892-911.

32. Tan $\mathrm{K}$, Zhang Z, Miao $\mathrm{K}$, et al. Dynamic integrated analysis of DNA methylation and gene expression profiles in in vivo and in vitro fertilized mouse post-implantation extraembryonic and placental tissues. Mol Hum Reprod. 2016; 22(7): 485-98.

33. Audic S, Claverie JM. The significance of digital gene expression profiles. Genome Res. 1997; 7(10): 986-95.

34. Karp NA, Spencer M, Lindsay H, et al. Impact of replicate types on proteomic expression analysis. J Proteome Res. 2005; 4(5): 1867-71.

35. Ren L, Zhang C, Tao L, et al. High-resolution profiles of gene expression and DNA methylation highlight mitochondrial modifications during early embryonic development. J Reprod Dev. 2017; 63(3): 247-61.

36. Brown JR, Phongthachit C, Sulkowski MJ. Immunofluorescence and image analysis pipeline for Drosophila motor neurons. Biol Methods Protoc. 2019; 4(1): bpz010.

37. Farthing CR, Ficz G, Ng RK, et al. Global mapping of DNA methylation in mouse promoters reveals epigenetic reprogramming of pluripotency genes. PLoS Genet. 2008; 4(6): e1000116.

38. Meissner A, Mikkelsen TS, Gu H, et al. Genome-scale DNA methylation maps of pluripotent and differentiated cells. Nature. 2008; 454(7205): 766-70.

39. Auclair G, Guibert S, Bender A, et al. Ontogeny of CpG island methylation and specificity of DNMT3 methyltransferases during embryonic development in the mouse. Genome Biol. 2014; 15(12): 545.

40. Canovas S, Ivanova E, Romar R, et al. DNA methylation and gene expression changes derived from assisted reproductive technologies can be decreased by reproductive fluids. Elife. 2017; 6: e23670.

41. Ficz G, Hore TA, Santos F, et al. FGF signaling inhibition in ESCs drives rapid genome-wide demethylation to the epigenetic ground state of pluripotency. Cell Stem Cell. 2013; 13(3): 351-9.

42. Onuma Y, Higuchi K, Aiki Y, et al. A stable chimeric fibroblast growth factor (FGF) can successfully replace basic FGF in human pluripotent stem cell culture. PLoS One. 2015; 10(4): e0118931.

43. Lanner F, Lee KL, Sohl M, et al. Heparan sulfation-dependent fibroblast growth factor signaling maintains embryonic stem cells primed for differentiation in a heterogeneous state. Stem Cells. 2010; 28(2): 191-200.

44. Kang M, Piliszek A, Artus J, et al. FGF4 is required for lineage restriction and salt-and-pepper distribution of primitive endoderm factors but not their initial expression in the mouse. Development. 2013; 140(2): 267-79.

45. Kosaka N, Sakamoto H, Terada M, et al. Pleiotropic function of FGF-4: its role in development and stem cells. Dev Dyn. 2009; 238(2): 265-76.

46. Feldman B, Poueymirou W, Papaioannou VE, et al. Requirement of FGF-4 for postimplantation mouse development. Science. 1995; 267(5195): 246-9.

47. Petell CJ, Alabdi L, He M, et al. An epigenetic switch regulates de novo DNA methylation at a subset of pluripotency gene enhancers during embryonic stem cell differentiation. Nucleic Acids Res. 2016; 44(16): 7605-17.

48. Sinkkonen L, Hugenschmidt T, Berninger P, et al. MicroRNAs control de novo DNA methylation through regulation of transcriptional repressors in mouse embryonic stem cells. Nat Struct Mol Biol. 2008; 15(3): 259-67. 
49. Jinawath A, Miyake S, Yanagisawa Y, et al. Transcriptional regulation of the human DNA methyltransferase $3 \mathrm{~A}$ and $3 \mathrm{~B}$ genes by $\mathrm{Sp} 3$ and $\mathrm{Sp} 1$ zinc finger proteins. Biochem J. 2005; 385(Pt 2): 557-64.

50. Zhu P, Guo H, Ren $Y$, et al. Single-cell DNA methylome sequencing of human preimplantation embryos. Nat Genet. 2018; 50(1): 12-19.

51. Guenatri M, Duffié R, Iranzo J, et al. Plasticity in Dnmt3L-dependent and -independent modes of de novo methylation in the developing mouse embryo. Development. 2013; 140(3): 562-72.

52. Watanabe D, Uchiyama K, Hanaoka K. Transition of mouse de novo methyltransferases expression from Dnmt3b to Dnmt3a during neural progenitor cell development. Neuroscience. 2006; 142(3): 727-37.

53. Chamberlain AA, Lin M, Lister RL, et al. DNA methylation is developmentally regulated for genes essential for cardiogenesis. J Am Heart Assoc. 2014; 3(3): e000976.

54. Li E, Bestor TH, Jaenisch R. Targeted mutation of the DNA methyltransferase gene results in embryonic lethality. Cell. 1992; 69(6): 915-26.

55. Sui L, An L, Tan K, et al. Dynamic proteomic profiles of in vivo- and in vitroproduced mouse postimplantation extraembryonic tissues and placentas. Biol Reprod. 2014; 91(6): 155.

56. Ptak GE, D'Agostino A, Toschi P, et al. Post-implantation mortality of in vitro produced embryos is associated with DNA methyltransferase 1 dysfunction in sheep placenta. Hum Reprod. 2013; 28(2): 298-305.

57. Wright K, Brown L, Casson P, et al. ART conditions are associated with global genomic hypomethylation at the blastocyst stage in mice. Fert Steril. 2008; $90 \mathrm{~S} 345$.

58. Yamanaka Y, Lanner F, Rossant J. FGF signal-dependent segregation of primitive endoderm and epiblast in the mouse blastocyst. Development 2010; 137(5): 715-24.

59. Goldin SN, Papaioannou VE. Paracrine action of FGF4 during periimplantation development maintains trophectoderm and primitive endoderm. Genesis. 2003; 36(1): 40-7.

60. Lu C-W, Yabuuchi A, Chen L, et al. Ras-MAPK signaling promotes trophectoderm formation from embryonic stem cells and mouse embryos. Nat Genet. 2008; 40(7): 921-6.

61. Li Z, Theus MH, Wei L. Role of ERK $1 / 2$ signaling in neuronal differentiation of cultured embryonic stem cells. Dev Growth Differ. 2006; 48(8): 513-23.

62. Nichols J, Silva J, Roode M, et al. Suppression of Erk signalling promotes ground state pluripotency in the mouse embryo. Development. 2009; 136(19): 3215-22.

63. Ito S, D'Alessio AC, Taranova OV, et al. Role of Tet proteins in $5 \mathrm{mC}$ to $5 \mathrm{hmC}$ conversion, ES-cell self-renewal and inner cell mass specification. Nature. 2010; 466(7310): 1129-33

64. Kang J, Lienhard M, Pastor WA, et al. Simultaneous deletion of the methylcytosine oxidases Tet1 and Tet3 increases transcriptome variability in early embryogenesis. Proc Natl Acad Sci U S A. 2015; 112(31): E4236-45.

65. Barrera $\mathrm{AD}$, García EV, Hamdi $\mathrm{M}$, et al. Embryo culture in presence of oviductal fluid induces DNA methylation changes in bovine blastocysts. Reproduction. 2017; 154(1): 1-12.

66. Chronopoulou E, Harper JC. IVF culture media: past, present and future. Hum Reprod Update. 2014; 21(1): 39-55 